\title{
Comparing the mechanical performance of synthetic and natural cellular materials
}

\author{
F.A.O. Fernandes, R.T. Jardin, A.B. Pereira, R.J. Alves de Sousa* \\ TEMA Centre for Mechanical Technology and Automation, Department of Mechanical Engineering, University of Aveiro, Campus de Santiago, $3810-193$ Aveiro, Portugal
}

\section{A R T I C L E I N F O}

\section{Article history:}

Received 13 May 2015

Revised 27 May 2015

Accepted 3 June 2015

Available online 20 June 2015

\section{Keywords:}

Cellular materials

Agglomerated cork

Expanded cork

Polymeric foams

Multiple impact

Dynamic loading

Energy absorption

Sustainable materials

\begin{abstract}
A B S T R A C T
This work compares the mechanical performance of agglomerated cork against synthetic materials typically used as impact energy absorbers. Particularly, the study will focus on the expanded polystyrene (EPS) and expanded polypropylene (EPP).

Firstly, quasi-static compression tests are performed in order to assess the energy storage capacity and to characterize the stress-strain behavior cellular materials under study. Secondly, guided drop tests are performed to study the response of these materials when subjected to multiple dynamic loading (two impacts). Thirdly, finite element analysis (FEA) is carried out in order to simulate the compressive behavior of the studied materials under dynamic loading.

Results show that agglomerated cork is an excellent alternative to the synthetic materials. Not only for being a natural and sustainable material but also for withstanding considerable impact energies. In addition, its capacity to keep some of its initial properties after loading (regarding mechanical properties and dimensions) makes this material highly desirable for multiple-impact applications.
\end{abstract}

(ㄷ) 2015 Elsevier Ltd. All rights reserved.

\section{Introduction}

Synthetic and natural cellular materials have been used in many applications. From packaging of goods to military devices, from civil to aerospace engineering, these materials have been used in engineering applications where a good energy absorption capability is a desired feature. These materials are also commonly used in applications such as thermal-acoustic insulation [1].

Cellular materials are the material of election also for personal protective devices where the best example can be found on head protection systems such as road-helmets [2-5]. In fact, under compressive loading, these materials can undergo large strain deformation while maintaining a low stress plateau before reaching densification. This behavior allows them to absorb large amounts of energy under low stresses. Fig. 1 shows a typical compressive stress-strain curve for cellular materials.

Expanded polystyrene (EPS), expanded polypropylene (EPP), cork or even metal foams are examples of these materials. The best material for each application depends on the application itself, depending on the mechanical loading, strain rate, etc. The material's mechanical behavior depends on the density, loading strain rate and it is also affected by the manufacturing process. This

\footnotetext{
* Corresponding author.

E-mail address: rsousa@ua.pt (R.J. Alves de Sousa).
}

dependency attracted many researchers trying to characterize those materials under quasi-static and dynamic loading [1,6-10].

EPS is possibly the most common within these materials, mainly due to a convenient cost-benefit ratio [1], being widely employed in the packaging industry. It is also employed in highly demanding applications such as impact absorption in safety gear. This closed cell foam absorbs energy by crushing mechanisms (collapse of walls). The EPS density is an important property because the yielding stress at which the foam crushes is directly related to it [11]. This parameter influences the EPS energy absorption capability, being responsible for the basic mechanisms of deformation and failure, determining the maximum crushing [1]. The typical stress-strain curve of EPS under compression is similar to the one illustrated in Fig. 1. In this, three regions can be identified: at very low stresses the material presents an almost linear elastic behavior, followed by a wide plateau where the stress remains almost constant, which leads to densification, where stress rises steeply for large strains.

Although this type of foam has an excellent first impact performance, in case of a subsequent impact in the same area, the protection level offered by EPS is minimal since the material deforms permanently without elastic recovery [7,12-14]. Thus, its energy absorption capability is significantly decreased after one impact, particularly in high-energy ones where large strains are reached. In order to overcome this issue, some materials were proposed 


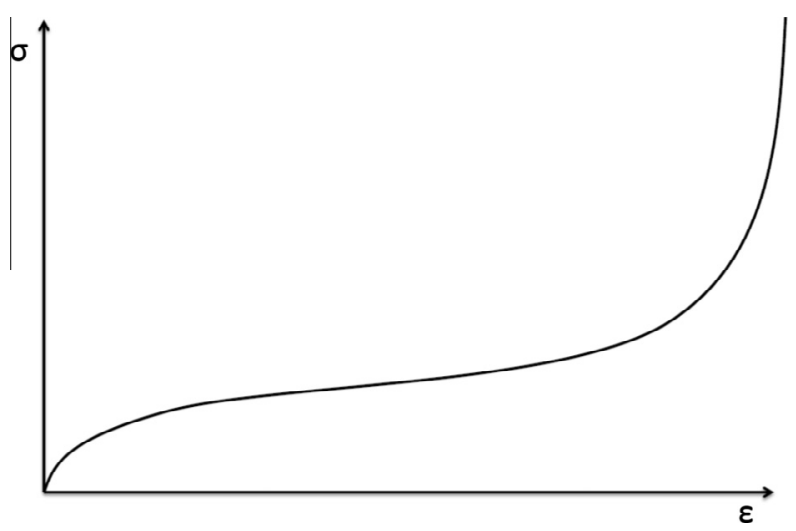

Fig. 1. Typical compressive stress-strain curve for cellular materials.

for multi-impact applications, such as EPP [7] and agglomerated cork $[2,3]$ as motorcycle helmet liners.

EPP is also a synthetic material like EPS. For a first impact, their mechanical behavior is quite similar [7]. Nevertheless, EPP foam has a multi-impact protection performance [7], as showing a relevant counterpart of elastic deformations. On the other hand, the quasi-static mechanical properties of EPP foams are attractive and available in recent publications, but impact properties are very limited [15].

Cork (in natural or agglomerated versions) is a natural cellular material capable of absorbing considerable amounts of energy $[16,7]$. Cork is characterized by having both a good energy absorption capacity and a high viscoelastic return (deforms mainly elastically). After one impact, the capacity of this material to keep absorbing energy is almost unchanged. Few researchers recently studying this material also tried to employ it in a great variety of applications, such as road helmets [2,3] and vehicle's passive safety mechanisms [18]. When compared to synthetic cellular materials, cork also appears as a sustainable alternative, once it is fully recyclable and the tree is not harmed as renewing its outer bark every nine years.

Nevertheless, cork is a complex natural cellular material with unknown or not well understood properties [19]. However, many researchers have extensively studied the fundamental aspects of cork's mechanical behavior under quasi-static axial compressive loading [20-27]. More recently, and regarding agglomerated cork (details on how agglomerated cork is produced can be found in [28]), the influence of cork density on cork's mechanical behavior under compression, as well as the subsequent recovery of dimensions were studied by Anjos et al. [29]. However, few researchers studied agglomerated cork's mechanical behavior when subjected to dynamic compressions. Gameiro et al. [19] studied cork's (natural and agglomerated) mechanical behavior under impact loading at strain rates ranging from 200 to $600 \mathrm{~s}^{-1}$. Nevertheless, the recovery dimensions at dynamic rates were not studied. In addition, quasi-static and dynamic tests were performed on agglomerated cork samples by Fernandes et al. [6] and the impacts on cork samples were simulated using finite element analysis (FEA), including the material's compression and relaxation.

The main objective of this study is the comparison of the mechanical response of EPS, EPP, agglomerated cork and expanded cork under multiple dynamic compressive loading (two impacts). There is also interest on the study of expanded cork and on evaluating its suitability as impact energy absorber, since there is no information about it in the literature. In addition, the impacts carried out experimentally were simulated for both agglomerated cork and expanded cork and also for EPS and EPP.

\section{Materials and methods}

In this study, EPS, EPP, agglomerated cork (AC) and expanded cork (EC) samples were tested. Expanded cork is different from the agglomerated one, mainly because of the manufacture process, which involves expansions under heat, pressure, and water addition. As a result, grain size is dramatically increased, density decreases and no binders are involved. Suberin (a subproduct of cork) acts as binder and the material is a $100 \%$ natural, in opposition to typical agglomerated cork that includes polyurethane as binder.

EPS and EPP were tested because they are among the most popular synthetic foams employed in energy absorption applications. Thus, it is possible to carry a comparison between the most used synthetic materials in energy absorption applications and cork solutions.

In order to perform this comparison, compression tests were performed at quasi-static and dynamic strain rates. The latter are guided impact tests, using a drop tower. Regarding the numerical simulations, Abaqus FE code was used to simulate the impacts.

\subsection{Materials}

In order to compare synthetic and natural cellular materials, EPS with a density value of $90 \mathrm{~kg} / \mathrm{m}^{3}$ and EPP with densities of 60 and $90 \mathrm{~kg} / \mathrm{m}^{3}$ were chosen (Fig. 2a). These are the density values commonly found in protective helmets. Regarding the cork samples (Fig. 2b), two densities were tested for AC, with $199 \mathrm{~kg} / \mathrm{m}^{3}$ and $216 \mathrm{~kg} / \mathrm{m}^{3}$, and one for EC with $159 \mathrm{~kg} / \mathrm{m}^{3}$.

The samples were produced by Petibol (EPP and EPS), Sofalca (EC) and CORKSRIBAS (AC), all of them Portuguese companies.

\subsection{Experimental tests}

Quasi-static and impact tests were performed in order to characterize and compare the materials for different strain rates. The procedure and setup of both tests is described below.

\subsubsection{Quasi-static compression tests}

Uniaxial quasi-static compressive tests were carried out using a Shimadzu AG50 KN testing machine with a video extensometer apparatus (Messphysic ME46NG).

The uniaxial compression test proceeded up until a $6.5 \mathrm{MPa}$ stress was achieved. At this value, it is possible to observe densification in agglomerated cork and synthetic foams.

The samples were cubes of an average size of $60 \times 60 \times 60 \mathrm{~mm}$. These samples were compressed at a velocity of $5 \mathrm{~mm} / \mathrm{min}$. The output force-displacement curves allowed to compute the Young moduli and energy absorbed per volume and to plot the stressstrain curve when compressed at quasi-static strain rates.

\subsubsection{Impact tests}

The impact tests were performed in a drop tower designed by the authors. This test rig consists in a 3 meter-high tube, which guides the hemispherical impactor. The impactor reaches an average impact speed of $4.5 \mathrm{~m} / \mathrm{s}$ (ranging between 4.3 and $4.7 \mathrm{~m} / \mathrm{s}$ ). This steel impactor has a diameter of $94 \mathrm{~mm}$ and weighs $5 \mathrm{~kg}$.

In order to measure the acceleration history during the impact, a uniaxial accelerometer (1201 Measurement Specialties) was placed inside the impactor. In addition, near the impact zone, there are two reflective object sensors (OPB700ALZ). These are separated from each other $15 \mathrm{~mm}$ in order to measure the impact speed. The signal from both reflective sensors and the accelerometer are acquired by an acquisition card TD 9816 at an acquisition rate of $2000 \mathrm{~Hz}$. The acceleration history and the speed values were 

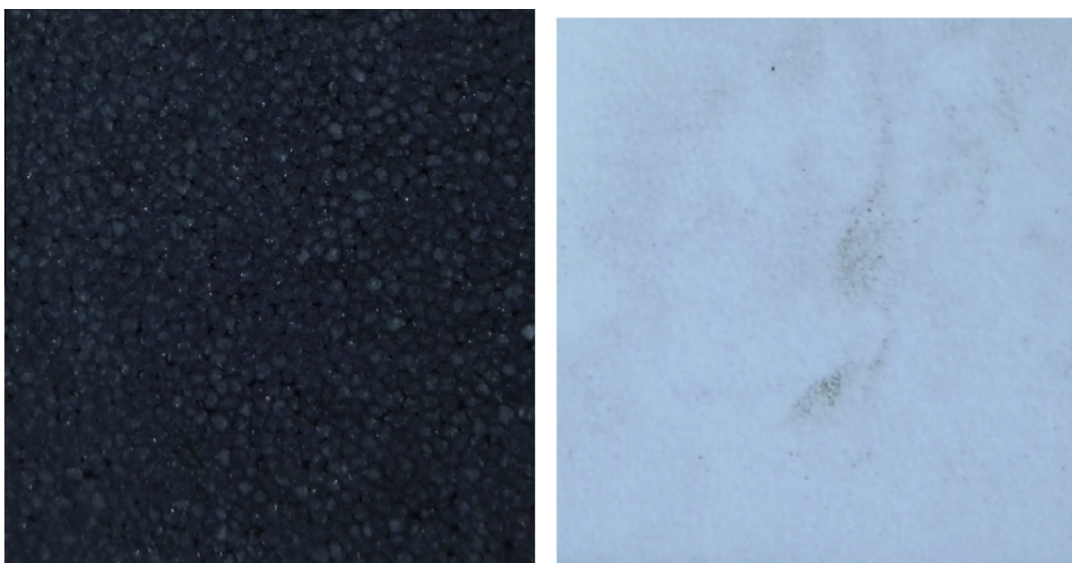

Fig. 2a. Samples: of EPP (black) and EPS (white). No magnification.
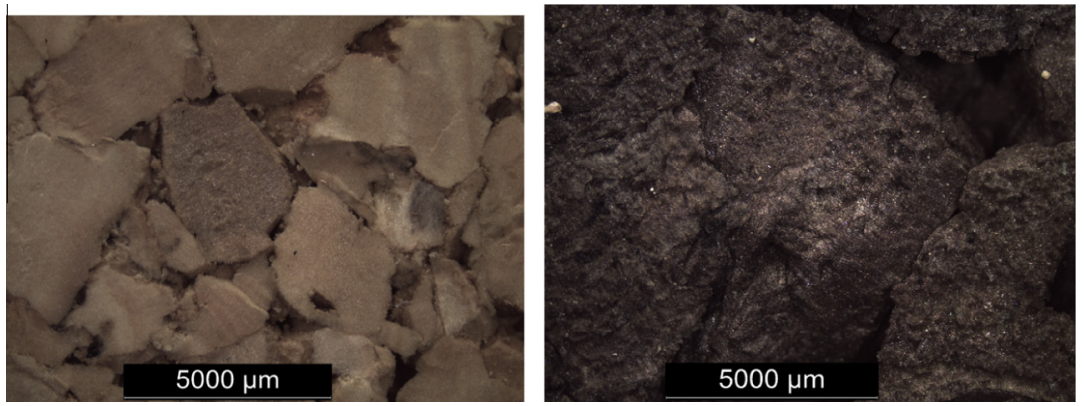

Fig. 2b. Samples of agglomerated and expanded (black) cork.

computed by a software developed in [6] using MATLAB programming language.

Fig. 3 shows in detail the impact area, where the samples are positioned on a steel block. The rectangular samples dimensions are $120 \times 120 \times 25 \mathrm{~mm}$, where the minor dimension corresponds to the samples thickness. The second impact is carried out approximately $30 \mathrm{~s}$ after the first impact.

The main goal of this test is the comparison of the agglomerated cork and the referred synthetic materials behavior under dynamic

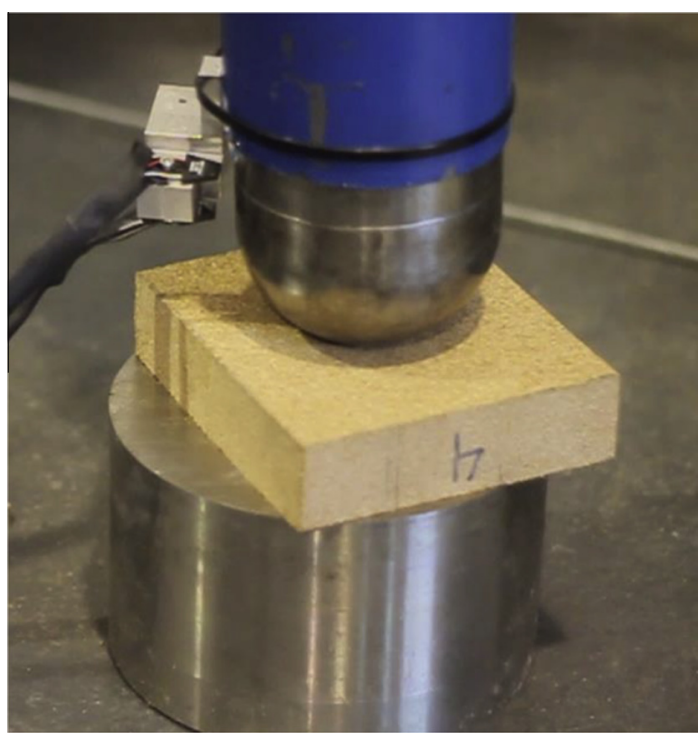

Fig. 3. Guide drop test apparatus [6]. loading. In other words, the capacity of these materials to absorb energy when subjected to dynamic loading.

\subsection{Finite element analysis}

The double impacts performed in the last section were simulated using Abaqus FE code Explicit solver. The samples dimensions' such as the impactor's mass, geometry and dimensions as well as the impact velocity match the experimental data. Two rigid parts were modeled, a fully constrained bottom plate that represents a steel base and half a sphere that represents the steel impactor. The samples were modeled as deformable bodies. The interaction between the sample and the rigid bodies was modeled with a hard surface-to-surface type of contact (Explicit). A friction coefficient of 0.2 was used to model such interaction. The rigid impactor has just one degree of freedom (vertical), whereas samples have no lateral constraint, as in the experiments. The setup is shown in Fig. 4

In order to create the FE model, the sample was modeled with eight-node fully integrated linear brick elements (Abaqus's C3D8 element). The rigid plate was modeled with rigid quadrangular elements (Abaqus's R3D4 element). The hemispherical impactor was modeled with rigid triangular elements (Abaqus's R3D3). More details about the mesh are presented in Table 1.

The meshes of each part were created avoiding distorted and warped elements. Special attention was given to elements size, in order to have reliable results but at the same time to have a reasonable computational time. Regarding the sample's mesh, several simulations were performed, increasing the number of elements until the results converged, defining the optimal number of elements. 


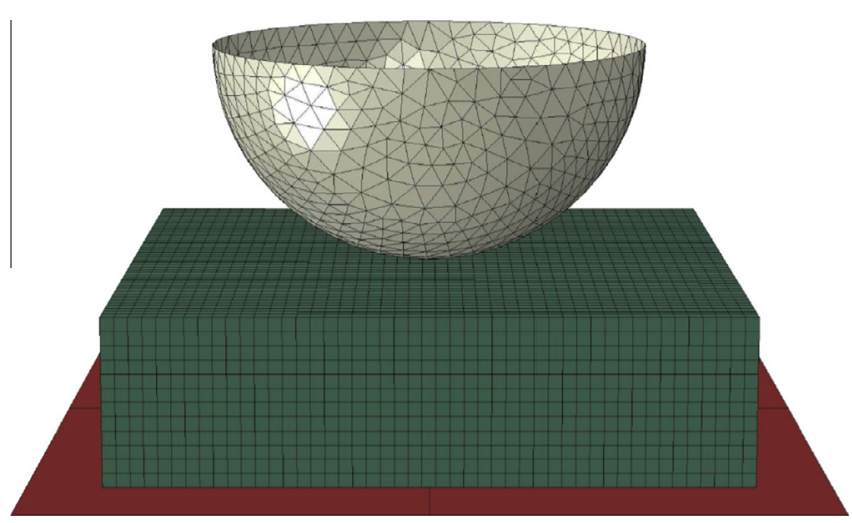

Fig. 4. Guided drop test - numerical setup [6].

Table 1

Mesh properties.

\begin{tabular}{lllrr}
\hline Part & Element type & $\begin{array}{l}\text { Abaqus } \\
\text { element }\end{array}$ & $\begin{array}{l}\mathrm{N}^{\circ} \text { of } \\
\text { elements }\end{array}$ & $\begin{array}{r}\mathrm{N}^{\circ} \text { of } \\
\text { nodes }\end{array}$ \\
\hline $\begin{array}{l}\text { Hemispherical } \\
\text { impactor }\end{array}$ & Rigid triangular shell & R3D3 & 1142 & 602 \\
$\begin{array}{l}\text { Bottom plate } \\
\text { Sample }\end{array}$ & $\begin{array}{l}\text { Rigid quadrangular shell } \\
\text { Eight-node linear brick }\end{array}$ & $\begin{array}{l}\text { R3D4 } \\
\text { C3D8 }\end{array}$ & $\begin{array}{r}17424 \\
20250\end{array}$ \\
\hline
\end{tabular}

\subsubsection{Material modeling}

Agglomerated cork and EPP were modeled as nonlinear hyperelastic materials. This simplification was considered valid due to the very small amount of plasticity observed experimentally. This was also verified in Fernandes et al. [6]. Agglomerated cork exhibits predominantly an elastic behavior, where the plastic behavior represents a small part of cork mechanical characteristics, only reached at very high deformations and high strain energies. On the other hand, EPS was modeled as a nonlinear plastic material.

As in Fernandes et al. [6], agglomerated cork was modeled with the combination between Hyperfoam and Mullins Effect material models. The same combination of material models was used to simulate EPP mechanical behavior under compression. Parameters were optimized for each material.

Hyperfoam is an isotropic and nonlinear material model typically used to characterize elastomeric foams that present hyperelastic behavior. It is also intended for finite-strain applications where it can deform elastically to large strains, up to $90 \%$ strain in compression.

In the Hyperfoam material model, the elastic behavior of the foams is based on the following strain energy function:

$\widetilde{U}=\sum_{i=1}^{N} \frac{2 \mu_{i}}{\alpha_{i}^{2}}\left[\lambda_{1}^{\alpha_{i}}+\lambda_{2}^{\alpha_{i}}+\lambda_{3}^{\alpha_{i}}-3+\frac{1}{\beta_{i}}\left(J^{-\alpha_{i} \beta_{i}}-1\right)\right]$

where $N$ is an integer (the polynomial order), $\lambda_{i}$ are the principal stretches, $J$ is the elastic volume ratio $\left(J=\lambda_{1} \lambda_{2} \lambda_{3}\right), \mu_{i}$ are shear moduli, $\alpha_{i}$ and $\beta_{i}$ are curve-fitting non-integral exponents.

The principal stretches, $\lambda_{i}$, are related to the principal nominal strains, $\varepsilon_{i}$, by:

$\lambda_{i}=1+\varepsilon_{i}$

In order to correctly model the permanent energy dissipation and stress softening effects in agglomerated cork, the Mullins Effect model is used together with the Hyperfoam material model, providing an extension to the isotropic elastomeric foam model. Thus, this material model is used to include the damage present in elastomeric foams, modeling energy absorption in foam components subjected to dynamic loading, under deformation rates that are high when compared to the characteristic foam time relaxation. The energy dissipation effects are accounted for by introducing an augmented strain energy density function of the form:

$U\left(\lambda_{i}, \eta\right)=\eta \widetilde{U}\left(\lambda_{i}\right)+\phi(\eta)$

where $\lambda_{i}(i=1,2,3)$ represent the principal mechanical stretches and $\tilde{U}\left(\lambda_{i}\right)$ is the strain energy potential for the primary foam behavior described by Eq. (1). The function $\phi(\eta)$ is a continuous function of the damage variable, $\eta$, and related to the damage function [30].

The model also predicts energy dissipation under purely volumetric deformation.

The damage variable, $\eta$, varies with the deformation according to:

$\eta=1-\frac{1}{r} \operatorname{erf}\left(\frac{U^{m}-\widetilde{U}}{m+\beta U^{m}}\right)$

where $U^{m}$ is the maximum value of $\tilde{U}$ at a material point during its deformation history; $r, \beta$ and $m$ are material parameters and $\operatorname{erf}(x)$ is the error function. While the parameters $r$ and $\beta$ are dimensionless, the parameter $m$ has the dimensions of energy.

The EPS foam was modeled as elasto-plastic material, where the elastic behavior of EPS is modeled with Hooke's law. To simulate the EPS plastic behavior, the Crushable Foam material model was employed. This model is based on the uniaxial-compressive response of closed-cell polymer foams given by:

$\sigma_{c}=\sigma_{c 0}+\frac{P_{0} \varepsilon}{1-\varepsilon-R}$

where $\sigma_{c}$ is the engineering compressive stress, $\sigma_{c 0}$ is the compressive yield stress, $P_{0}$ is the effective gas pressure in the cells, and $R$ is the foam relative density (foam density divided by solid polymer density). This same strategy was used by Fernandes et al. [5] to model the behavior of EPS under compression.

The quasi-static compression curves presented in Fig. 5 were used as input data. The strain energy potential order, $N$, used in

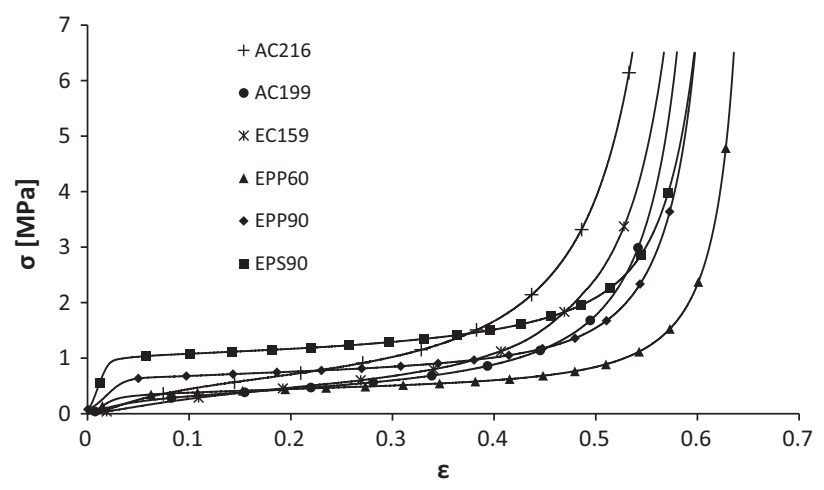

Fig. 5. Synthetic foams and agglomerated cork stress-strain curves.

Table 2

Material parameters introduced in Abaqus to characterize agglomerated cork, EPP and EPS.

\begin{tabular}{lllllllll}
\hline Material & $\rho\left(\mathrm{kg} / \mathrm{m}^{3}\right)$ & $E(\mathrm{MPa})$ & $v$ & $p_{t} / p_{c 0}$ & $\sigma_{c 0} / p_{c 0}$ & $r$ & $m$ & $\beta$ \\
\hline AC & 199.1 & - & 0 & - & - & 1.8 & 0.01 & 0.1 \\
& 216.2 & - & 0.3 & - & - & 1.1 & 0.5 & 0.1 \\
EC & 159.4 & - & 0 & - & - & 1.01 & 0.3 & 0.1 \\
EPP & 60 & - & 0.04 & - & - & 1.01 & 0.01 & 0.1 \\
& 90 & - & 0.03 & - & - & & & \\
EPS & 90 & 44 & 0 & 0.1 & 2.5 & - & - & - \\
\hline
\end{tabular}


Hyperfoam material model was 3 . This was concluded as the best value to model agglomerated cork and EPP. These and other important material parameters introduced in the material models are presented in Table 2. In this, the ratios $p_{t} / p_{c 0}$ and $\sigma_{c 0} / p_{c 0}$ are the hydrostatic and compression yield stress ratios respectively.

\section{Results and discussion}

\subsection{Quasi-static tests}

Fig. 5 presents the average stress-strain curve obtained from testing 5 samples of each material. As expected, these cellular materials exhibit a steep increase of stress for very small strains $(\approx 5 \%)$. Then, these materials exhibit a wide plateau $(\approx 5 \%$ to $60 \%$ of strain), keeping a small stress. This plateau is the key responsible for the energy absorption capacity of cellular materials. Finally the materials reach densification (when cell walls finally collapse), characterized by an accentuated stress increase that occurs for a given small strain variation [11].

The uniaxial quasi-static stress-strain curves of all the materials tested can now be compared. From a first look, it can be stated that synthetic materials have a higher Young's modulus than agglomerated cork. This fact allows synthetic foams to achieve the plateau region with smaller deformations. Also, synthetic foams present a lower slope in plateau region and a densification for higher strain values, when compared to agglomerated cork. Once the goal is absorbing large amounts of energy, the mechanical response preferably should include a long plateau with moderate stress values, followed by densification only reached for high strains. From this point of view, and focusing only on the agglomerate cork, the AC216 is interesting because exhibits a high plateau stress. Nevertheless, it reaches densification sooner than the other agglomerates. Also, AC199 densificates only for high strains but the plateau region has low stress values. Finally, EC159 presents a commitment between a high stress plateau and densification at high strains. In the end, as already referred, the material choice will depend on the desired application and on the allowed stress level.

Regarding synthetic foams, they exhibit high plateaus stress and densification only for high strains. However, regarding multiple impacts, synthetic foams do not perform as well as agglomerated cork. This is demonstrated in the next section.

Fig. 7 shows the average value of Young's modulus and its dispersion. These values were measured experimentally by computing the slope in the linear elastic region. The most dominant factor of Young's moduli is the relative density [11]. This is clear by analyzing the agglomerated cork samples. Even the agglomerated cork with higher Young's modulus (AC216) has a small value compared with synthetic foams (Fig. 6).

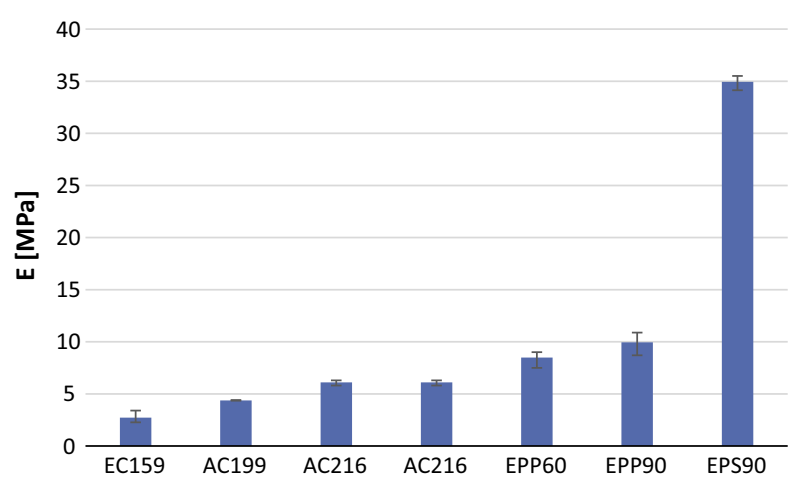

Fig. 6. Young Moduli of agglomerated cork and synthetic foams.

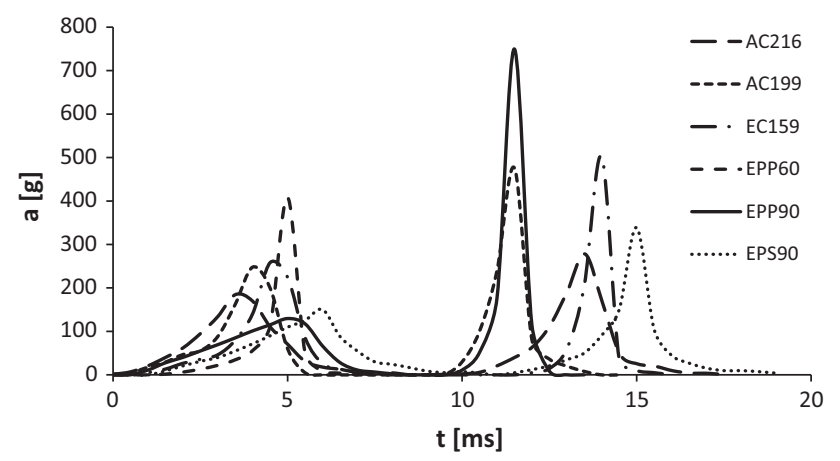

Fig. 7. Acceleration curves with $5 \mathrm{~kg}$ impactor.

Table 3

Mean acceleration peak increase in the second impact.

\begin{tabular}{ll}
\hline Sample & Mean acceleration peak increase (\%) \\
\hline AC216 & 34.3 \\
AC199 & 85.4 \\
EC159 & 37.1 \\
EPP60 & $-^{\mathrm{a}}$ \\
EPP90 & 491.5 \\
EPS90 & 114.6
\end{tabular}

a Did not withstand the second impact.

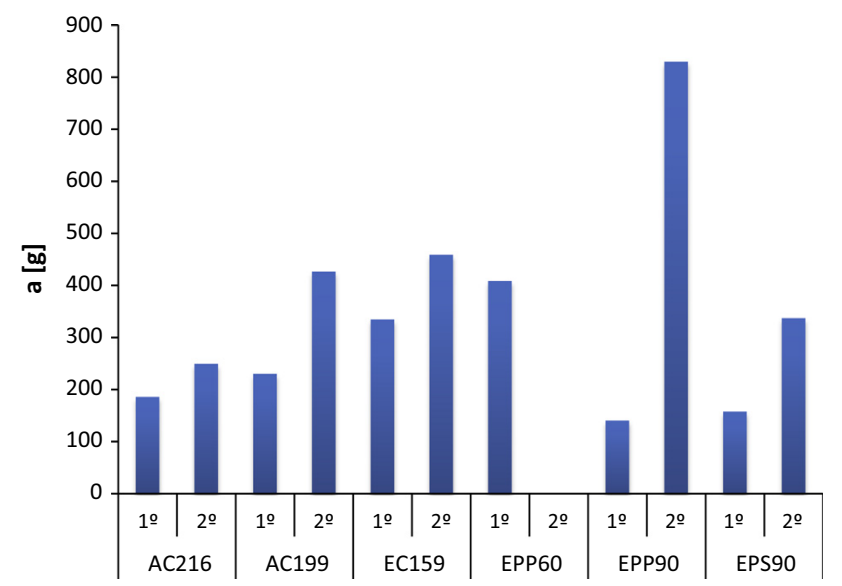

Fig. 8. Mean acceleration peak value for both impacts (EPP60 missing since it was destroyed after first impact).

\subsection{Dynamic tests}

Table 3 and Fig. 7 depict impact test for the analyzed materials. It is worth noting that the EPP60 s impact data is missing, since the first impact destroyed the samples.

Fig. 8 and Table 3 shows that synthetic materials clearly have a higher degradation of its properties than cork. It was possible to visually verify that EPP has a viscoelasticity similarly to agglomerated cork. However, this EPP feature does not bring any advantage over EPS, which has a perfectly plastic deformation outside the linear elastic zone.

Comparing the tested cork samples, it is possible to conclude that there is almost none degradation of the AC216 properties, even after 4 or 5 impacts. Further tests, with different masses showed that cork agglomerates can be employed in applications with a wide range of impact energies. Guided impact tests with a $10 \mathrm{~kg}$ impactor were also performed on agglomerated cork samples. In these tests, and for instance, AC199 agglomerate had a 


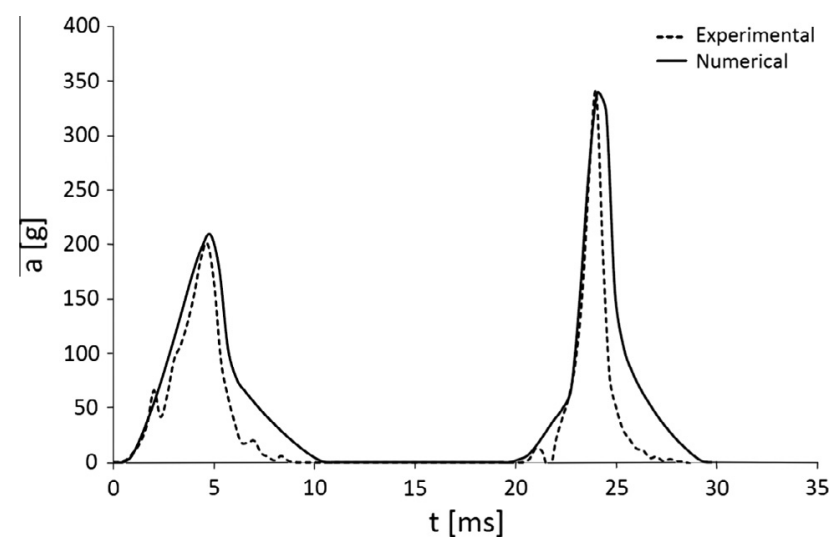

Fig. 9. Comparison between numerical and experimental results - AC199.

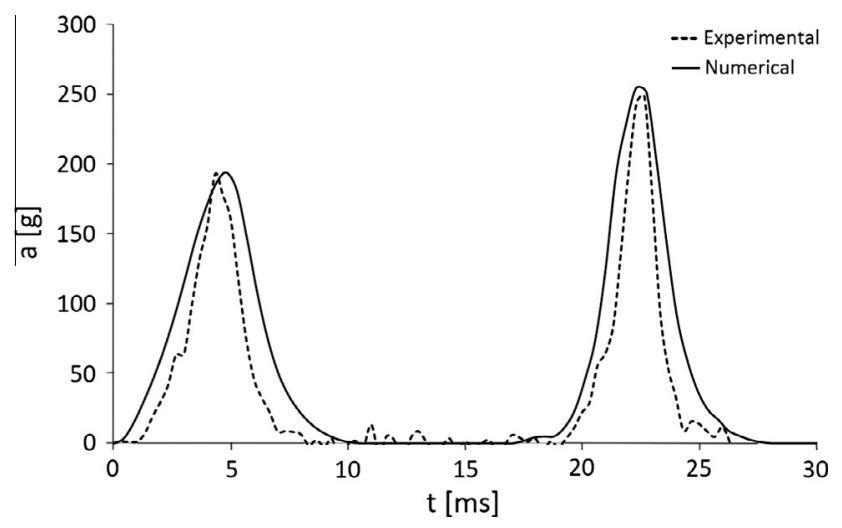

Fig. 10. Comparison between numerical and experimental results - AC216.

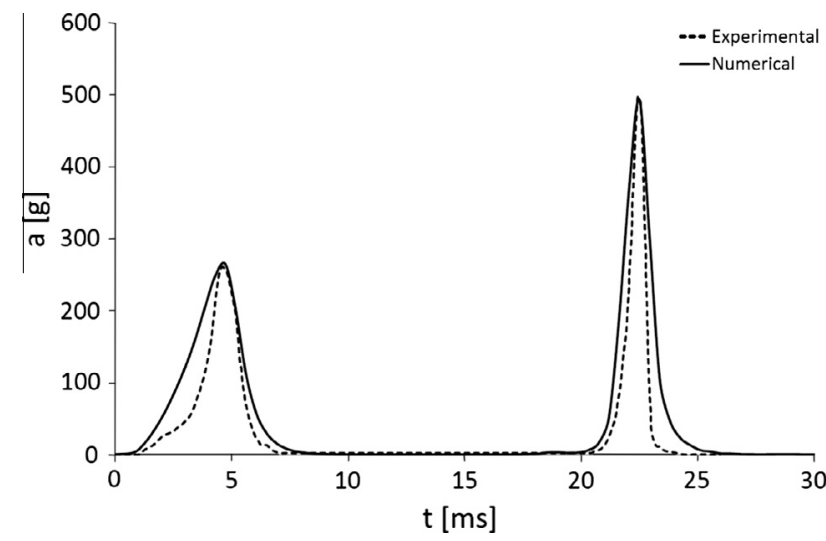

Fig. 11. Comparison between numerical and experimental results - EC159.

much reduced degradation of its properties, $1.7 \%$. Also, in both second impacts with a $5 \mathrm{~kg}$ and a $10 \mathrm{~kg}$ impactor, the peak acceleration values were approximately the same. In addition, the EC159 agglomerate presents a noticeable degradation of its properties, but with a good first impact performance. Thus, it can be concluded that the expanded agglomerated cork is the most suitable for low impact energies.

\subsection{Simulations}

The impact tests performed in this study were also simulated. This is important to make further tests saving material and time. The results from FEA were compared against the experiments.

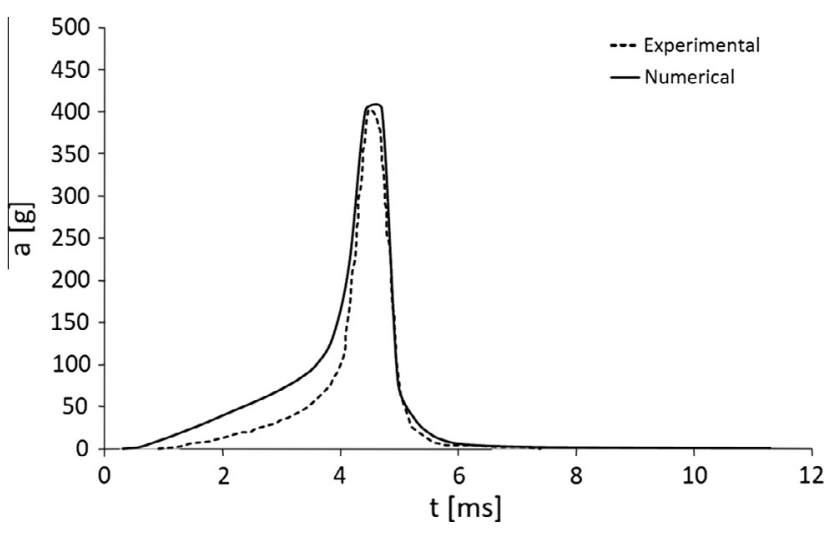

Fig. 12. Comparison between numerical and experimental results - EPP60.

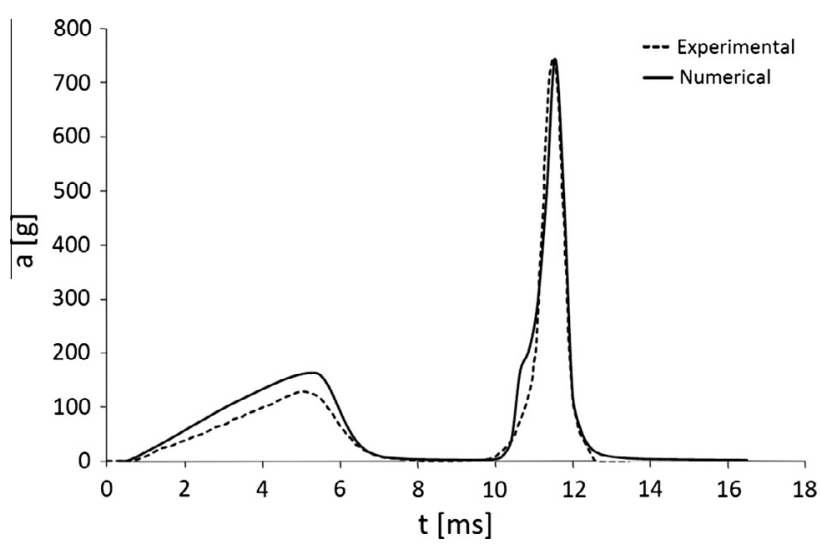

Fig. 13. Comparison between numerical and experimental results - EPP90.

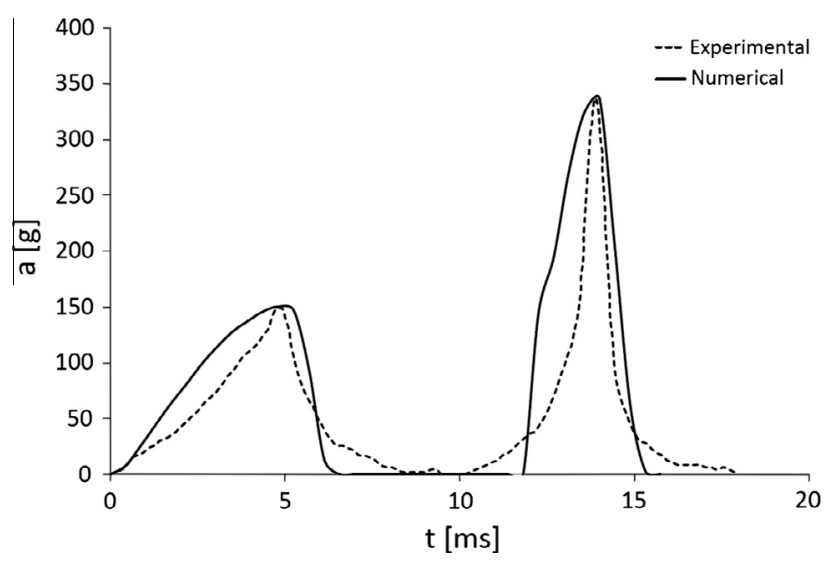

Fig. 14. Comparison between numerical and experimental results - EPS90.

Figs. 9-14 compare the impactor's uniaxial acceleration-time history for both natural and synthetic materials. Experimental results were filtered to eliminate small oscillations.

In general, the results obtained in FEA are close to the ones measured experimentally. The acceleration peaks in the simulations with AC199, AC216 and EC159 corks and with both EPP60 and EPP90 are very close to the ones measured experimentally. Among these referred materials, the worst approximation was obtained in the first impact with EPP90. Regarding the impacts duration in the simulations, generally these are very similar to the ones measured experimentally, being slightly higher in some simulations. Thus, as concluded in Fernandes et al. [6] the combination between Abaqus's Hyperfoam and Mullins Effect material 
models is capable of representing, with very good reliability, the behavior of materials such as agglomerated cork or EPP.

The results agreement were not so good in the simulation of EPS under double compression. The acceleration peaks of both impacts are reasonably represented. However, the numerical curve during both impacts is wider than the experimental. Nevertheless, the impact duration is approximately the same for both impacts. This means that higher energies are reached during the impacts in simulations with EPS.

\section{Conclusions}

With the increasing need for sustainable and recyclable materials, it becomes necessary to employ environmental friendly solutions even in demanding engineering applications.

Cork and its agglomerated versions are well-known materials in what concerns insulation applications, from thermal to acoustics. However, the capacity of this organic material to absorb impact energy is also noticeable.

This work focused on the comparison of two main types of agglomerated cork and two types of synthetic foams: the so-called white agglomerated cork, with smaller grains and higher densities and the expanded (black) cork, with larger grains and lower densities. Expanded polystyrene and expanded polypropylene were the chosen synthetic foams for comparison purposes.

Quasi-static and impact tests were performed. Results indicate a larger Young's modulus and higher plateau stresses for synthetic materials, which indicates more energy absorbed per unit volume under low stresses and quasi-static conditions. However, when impact situations are evaluated, natural materials show a much better compromise between performance and endurance under several impacts.

It should be noted that for instance helmet standards are evolving in the sense to require multiple impacts for validation purposes. This can be the definitive key (along with environmental aspects) to drive a higher usage of natural cellular materials like cork.

FEA were also performed, simulating the double impacts performed on all materials tested in this study. In general, the results obtained in FEA are close to the ones measured experimentally. The worst results were obtained in the simulation of impacts on EPS. In this sense, an accurate and reliable framework to simulate the mechanical response of natural and synthetic cellular materials was established.

The ultimate goal of this work is to infer the viability of replacing synthetic foams by natural ones. From the results and comparisons herein shown, it appears to be a completely plausible (if not better) solution for protective liners.

\section{Acknowledgements}

Support given by the Portuguese Science Foundation (FCT), Grant PTDC/EME-TME/109856 and COMPETE program and the scholarship SFRH/BD/91292/2012 are deeply acknowledged. CORKSRIBAS, Petibol and Sofalca are acknowledged for supplying the materials samples.

\section{References}

[1] L. Di Landro, G. Sala, D. Olivieri, Deformation mechanisms and energy absorption of polystyrene foams for protective helmets, Polym. Test. 21 (2002) 217-228.

[2] R.J. Alves de Sousa, D.F.S. Gonçalves, R.M. Coelho, F.M.V.H. Teixeira-Dias, Assessing the effectiveness of the use of a natural cellular material as safety padding in motorcycle helmet simulation, Trans. Soc. Model. Simul. Int. 88 (5) (2012) 579-590.

[3] R.M. Coelho, R.J. Alves de Sousa, F.A.O. Fernandes, F.M.V.H. Teixeira-Dias, New composite liners for energy absorption purposes, Mater. Des. 43 (2013) 384392.

[4] F.A.O. Fernandes, R.J. Alves de Sousa, Motorcycle Helmets - A State of the Art Review, Accident Anal. Prevent. 56 (2013) 1-21.

[5] F.A.O. Fernandes, R.J. Alves de Sousa, Finite element analysis of helmeted oblique impacts and head injury evaluation with a commercial road helmet, Struct. Eng. Mech. 48 (5) (2013) 661-679.

[6] F.A.O. Fernandes, R.J.S. Pascoal, R.J. Alves de Sousa, Modelling impact response of agglomerated cork, Mater. Des. 58 (2014) 499-507.

[7] F.M. Shuaeib, A.M.S. Hamouda, S.V. Wong, R.S. Radin Umar, M.M.H. Megat Ahmed, A new motorcycle helmet liner material: the finite element simulation and design of experiment optimization, Mater. Des. 28 (2007) 182-195.

[8] N.J. Mills, R. Stämpfli, F. Marone, P.A. Brühwiler, Finite element micromechanics model of impact compression of closed-cell polymer foams, Int. J. Solids Struct. 46 (3-4) (2009) 677-697.

[9] S. Ouellet, D. Cronin, M. Worswick, Compressive response of polymeric foams under quasi-static, medium and high strain rate conditions, Polym. Test. 25 (6) (2006) 731-743.

[10] H.X. Zhu, N.J. Mills, Modelling the creep of open-cell polymer foams, J. Mech. Phys. Solids 147 (7) (1999) 1437-1457.

[11] L.J. Gibson, M.F. Ashby, Cellular Solids: Structure and Properties, second ed., Cambridge University Press, Cambridge, 1997.

[12] F.M. Shuaeib, A.M.S. Hamouda, M.M. Hamdan, R.S. Radin Umar, M.S.J. Hashmi, Motorcycle helmet: Part II, materials and design issues, J. Mater. Process. Technol. 123 (2002) 422-431.

[13] F.M. Shuaeib, A.M.S. Hamouda, Umar R. Radin, M.M. Hamdan, M.S.J. Hashmi, Motorcycle helmet, Part III, manufacturing issues, J. Mater. Process. Technol. 123 (2002) 432-439.

[14] A. Gilchrist, N.J. Mills, Modelling of the impact response of motorcycle helmets, Int. J. Impact Eng. 15 (3) (1994) 201-218.

[15] L.J. Gibson, M.F. Ashby, Cellular Solids structures and properties, second ed., Cambridge University Press, Cambridge, MA, 1997.

[16] I. Alcântara, F. Teixeira-Dias, M. Paulino, Cork composites for the absorption of impact energy, Compos. Struct. 95 (2013) 16-27.

[17] M. Paulino, F. Teixeira-Dias, An energy absorption performance index for cellular materials - development of a side-impact cork padding, Int. J. Crashworthiness 16 (2) (2011) 135-153.

[18] M. Paulino, F. Teixeira-Dias, On the Use of Polyurethane Foam Paddings to Improve Passive Safety in Crashworthiness Applications, in: E. Sharmin, F. Zafar (Eds.), Polyurethane, InTech, 2012, pp. 337-354.

[19] C.P. Gameiro, J. Cirne, G. Gary, V. Miranda, J. Pinho-da-Cruz, F. Teixeira-Dias, Numerical and experimental study of the dynamic behaviour of cork, Des. Use Light-Weight Mater. (2005) 65-84.

[20] O. Anjos, H. Pereira, M.E. Rosa, Effect of quality, porosity and density on the compression properties of cork, Holz als Roh-und Werkstoff 66 (4) (2008) 295-301.

[21] M.A. Fortes, M.T. Nogueira, The Poisson effect in cork, Mater. Sci. Eng. A 122 (1989) 227-232.

[22] M.A. Fortes, M.E. Rosa, H. Pereira, A cortiça, IST Press, 2004.

[23] L.J. Gibson, K.E. Easterling, M.F. Ashby, The structure and mechanics of cork, Proc. R. Soc. London A 377 (1981) 99-117.

[24] L.J. Gibson, M. Ashby, G.S. Schajer, C.I. Robertson, The mechanics of twodimensional cellular materials, Proc. R. Soc. London 382 (1982) 25-42.

[25] J. Mano, The viscoelastic properties of cork, J. Mater. Sci. 37 (2002) 257-263.

[26] H. Pereira, J. Graça, C. Baptista, The effect of growth-rate on the structure and compressive properties of cork, IAWA Bull. 13 (4) (1992) 389-396.

[27] M.E. Rosa, M.A. Fortes, Rate effects on the compression and recovery of dimensions of cork, J. Mater. Sci. 23 (3) (1988) 879-885.

[28] H. Pereira, Cork: Biology, Production and Uses, Elsevier, Amsterdam, 2007.

[29] A. Anjos, C. Rodrigues, J. Morais, H. Pereira, Effect of density on the compression behaviour of cork, Mater. Des. 53 (2014) 1089-1096.

[30] Abaqus 6.10 documentation. Hibbitt, Karlsson \& Sorensen, Inc., 2010. 